\title{
CLEAR EVIDENCE FOR THE PRESENCE OF SECOND-GENERATION ASYMPTOTIC GIANT BRANCH STARS IN METAL-POOR GALACTIC GLOBULAR CLUSTERS
}

\author{
D. A. García-Hernández ${ }^{1,2}$, Sz. Mészáros ${ }^{3}$, M. Monelli ${ }^{1,2}$, S. Cassisi ${ }^{1,2,4}$, P. B. Stetson ${ }^{5}$, O. Zamora ${ }^{1,2}$, \\ M. Shetrone ${ }^{6}$, and S. LuCATEllo ${ }^{7}$ \\ ${ }^{1}$ Instituto de Astrofísica de Canarias, C/Via Láctea s/n, E-38205 La Laguna, Spain; agarcia@iac.es, monelli@iac.es, ozamora@iac.es \\ ${ }^{2}$ Departamento de Astrofísica, Universidad de La Laguna (ULL), E-38206 La Laguna, Spain \\ ${ }^{3}$ ELTE Gothard Astrophysical Observatory, H-9704 Szombathely, Szent Imre herceg út, Hungary \\ ${ }^{4}$ INAF-Osservatorio Astronomico di Teramo, via M. Maggini, 64100, Teramo, Italy; cassisi@oa-teramo.inaf.it \\ ${ }^{5}$ Dominion Astrophysical Observatory, Herzberg Institute of Astrophysics, National Research Council, 5071 West Saanich Road, Victoria, BC V9E 2E7, Canada \\ ${ }^{6}$ University of Texas at Austin, McDonald Observatory, Fort Davis, TX 79734, USA \\ ${ }^{7}$ INAF-Osservatorio Astronomico di Padova, vicolo dell Osservatorio 5, I-35122 Padova, Italy \\ Received 2015 November 3; accepted 2015 November 17; published 2015 December 3
}

\begin{abstract}
Galactic globular clusters (GCs) are known to host multiple stellar populations: a first generation (FG) with a chemical pattern typical of halo field stars and a second generation (SG) enriched in $\mathrm{Na}$ and $\mathrm{Al}$ and depleted in $\mathrm{O}$ and $\mathrm{Mg}$. Both stellar generations are found at different evolutionary stages (e.g., the main-sequence turnoff, the subgiant branch, and the red giant branch (RGB)). The non detection of SG asymptotic giant branch (AGB) stars in several metal-poor $([\mathrm{Fe} / \mathrm{H}]<-1)$ GCs suggests that not all SG stars ascend the AGB phase, and that failed AGB stars may be very common in metal-poor GCs. This observation represents a serious problem for stellar evolution and GC formation/evolution theories. We report fourteen SG-AGB stars in four metal-poor GCs (M13, M5, M3, and M2) with different observational properties: horizontal branch (HB) morphology, metallicity, and age. By combining the H-band Al abundances obtained by the Apache Point Observatory Galactic Evolution Experiment survey with ground-based optical photometry, we identify SG Al-rich AGB stars in these four GCs and show that Al-rich RGB/AGB GC stars should be Na-rich. Our observations provide strong support for present, standard stellar models, i.e., without including a strong mass-loss efficiency, for low-mass HB stars. In fact, current empirical evidence is in agreement with the predicted distribution of FG and SG stars during the He-burning stages based on these standard stellar models.
\end{abstract}

Key words: globular clusters: general - globular clusters: individual (M13, M5, M3, M2) -

stars: AGB and post-AGB

\section{INTRODUCTION}

It is well known that all Galactic globular clusters (GCs) host multiple (at least two) stellar populations (see Gratton et al. 2012; Piotto et al. 2012 and references therein). This result has been deduced mainly from the general presence of the so-called $\mathrm{C}-\mathrm{N}, \mathrm{O}-\mathrm{Na}$, and $\mathrm{Mg}-\mathrm{Al}$ anticorrelations. First-generation (FG) stars display normal $\mathrm{Na}$ (and $\mathrm{Al}$ ) abundances (i.e., typical of halo field stars), while second-generation (SG) stars-which may have additional subpopulations-show $\mathrm{Na}$ (and $\mathrm{Al}$ ) enhancements. These SG additional subpopulations of stars are also characterized by He overabundances that change from cluster to cluster (e.g., Milone et al. 2014). The presence of FG and SG stars in GCs has been clearly traced-using both spectroscopic and/or photometric data-in the various evolutionary sequences, from the main sequence up to the more advanced evolutionary stages (e.g., Gratton et al. 2001; Carretta et al. 2009; Milone et al. 2012; Marino et al. 2014). However, as initially noticed by Norris et al. (1981; see also Gratton et al. 2010), there are claims concerning the paucity (or lack) of SG stars along the asymptotic giant branch (AGB) of some clusters. If confirmed, this occurrence would represent a challenge for stellar evolution and the formation and evolution models of these complex stellar systems (Charbonnel et al. 2013; Cassisi et al. 2014).

\footnotetext{
8 See also Johnson \& Pilachowski (2012) and Lapenna et al. (2015) for the non-detection of SG-AGBs in M13 (a twin of NGC 6752) and M62 (a slightly more metal-rich cluster with $[\mathrm{Fe} / \mathrm{H}] \approx-1.2$ ).
}

Recent spectroscopic observations of AGB stars in the metal-poor $([\mathrm{Fe} / \mathrm{H}] \approx-1.56)$ GC NGC 6752 (Campbell et al. 2013) have found no Na-rich SG-AGB stars in this cluster. ${ }^{8}$ Campbell et al. (2013) have explained these puzzling observations as due to the fact that all SG stars do not ascend the AGB (AGB-manqué stars). They suggest that a stronger mass-loss in SG horizontal branch (HB) stars could explain their observations, and that AGB-manqué stars may be very common in metal-poor $([\mathrm{Fe} / \mathrm{H}]<-1)$ Galactic GCs. ${ }^{9}$

The Na-poor nature of all AGB stars analyzed in NGC 6752 (also in M13 and M62) poses an apparent problem for stellar evolution. This difficulty arises because synthetic HB models of NGC 6752, based on canonical-i.e., without a strong massloss efficiency during the core He-burning stage - HB stellar models, do not predict the observed lack of SG-AGB stars. More recently, Cassisi et al. (2014) have critically discussed such a mass-loss scenario during the core He-burning stage. They show that the required mass-loss rates are much higher than any of the current theoretical and empirical constraints, and that if all SG HB stars do not climb the AGB it would be virtually impossible to reproduce the number ratio of AGB to HB stars (the $R_{2}$ parameter) in NGC 6752 and a few other clusters with similar/dissimilar observational properties. Thus,

\footnotetext{
9 Johnson et al. (2015) have very recently found SG (Na-rich) AGB stars in the massive and more metal-rich $([\mathrm{Fe} / \mathrm{H}] \approx-0.70) \mathrm{GC} 47$ Tuc but they argue that the high metallicity leads to a different HB morphology (predominantly red) with an insignificant population of hot $\mathrm{HB}$ stars and failed AGB stars, which likely make up the missing Na-rich AGB in NGC 6752 (and M13).
} 
at present there is no simple explanation for the apparent lack of SG-AGB stars in these metal-poor GCs.

Here we report SG-AGB stars in the GC M13-a twin of NGC 6752 - and another three GCs (M5, M3, and M2) of similar metallicity but with distinct observational properties in terms of HB morphology and age. For this, we combined the H-band abundances measured by the Apache Point Observatory Galactic Evolution Experiment (APOGEE; Majewski et al. 2015) and the most recent ground-based photometry of these GCs. The $\mathrm{C}$ and $\mathrm{N}$ abundances are significantly affected by the occurrence of both the first dredge-up during the early RGB and by non-canonical extra-mixing processes after the RGB bump (e.g., Cassisi \& Salaris 2013). Thus, for the present analysis we decided to analyze the abundances of $\mathrm{Mg}, \mathrm{Al}, \mathrm{Na}$, and $\mathrm{O}$ which are barely-if at all-affected by these mixing processes during the RGB and AGB stages in the evolution of low-mass stars.

\section{APOGEE DATA AND GROUND-BASED PHOTOMETRY}

The APOGEE survey observed ten northern GCs, covering a range of metallicity $[\mathrm{Fe} / \mathrm{H}]$ from -0.8 down to -2.4 , including cluster members with well-characterized stellar parameters and abundances from existing high-resolution optical spectra, as well as many additional cluster giant stars currently lacking such detailed abundances (Mészáros et al. 2015). The stellar parameters and chemical abundances of nine elements $(\mathrm{Fe}, \mathrm{C}$, $\mathrm{N}, \mathrm{O}, \mathrm{Mg}, \mathrm{Al}, \mathrm{Si}, \mathrm{Ca}$, and $\mathrm{Ti}$ ) for 428 cluster star members in these ten GCs have recently been reported by us (Mészáros et al. 2015). We used photometry and theoretical isochrones to constrain the effective temperature $\left(T_{\text {eff }}\right)$ and surface gravity $(\log g)$. We then used an independent semi-automated method for precise (up to the $\sim 0.1$ dex level) elemental abundance determination from the high-resolution $(\sim 22,500)$ and highquality (signal-to-noise $>70$ per pixel) $H$-band $(\sim 1.5-1.7 \mu \mathrm{m}$ ) spectra (Mészáros et al. 2015).

The APOGEE abundances are measured from neutral lines of $\mathrm{Fe}, \mathrm{Al}, \mathrm{Mg}$, etc.; the $H$-band single-ionized lines are not detected in metal-poor GC giants. The APOGEE $H$-band data offer several advantages with respect to previous optical spectroscopic studies of GC giants: (i) they enable us to analyze these ten clusters in a homogeneous way (covering almost the full extent of the RGB); (ii) nonlocal thermodynamic equilibrium (NLTE) effects on the spectral lines of neutral species such as $\mathrm{Fe}, \mathrm{Al}, \mathrm{Mg}$, etc. are less important than in the optical range because in the $H$-band these lines are formed deeper in the atmosphere (see Section 4); and (iii) the increased number of APOGEE stars compared to the literature permit us to discover more Al-rich stars, making the $\mathrm{Mg}-\mathrm{Al}$ plane clearer in the APOGEE data than in previous studies.

Ground-based $U, B, V, I$ photometry is available at this time for six of the ten GCs observed by APOGEE. The groundbased photometry is taken from the private collection by P. Stetson, which is based upon a large corpus of the most recent observations obtained mainly from public astronomical archives. Our $U$ and $B V I$ magnitudes are precise to the level of $<0.002$ and $<0.001 \mathrm{mag}$, respectively, and are probably accurate to the Landolt system to $<0.02$ and $<0.01$ mag on a star-by-star basis, for stars in the range $(11.5 \leqslant V \leqslant 15.5)$ of the APOGEE observations (see, e.g., Stetson et al. 2014).

\section{COLOR-MAGNITUDE DIAGRAMS AND THE $\left(V, C, C_{u, b, i}\right)$ PSEUDO-COLOR-MAGNITUDE DIAGRAM (CMD)}

We made use of the ground-based $U, B, V, I$ photometry mentioned above to construct several CMDs for each GC observed by APOGEE and separate the AGB from the RGB stars. ${ }^{10}$ We find that the combination of the $U-(U-I)$, $\mathrm{I}-(U-I)$, and $V-(B-I)$ CMDs gives an efficient RGB/ AGB separation (Figure 1; see below).

In Mészáros et al. (2015) we used an extreme-deconvolution (XD) method ${ }^{11}$ to identify $\mathrm{FG}$ and $\mathrm{SG}$ stars in the $\mathrm{Al}-\mathrm{Mg}$ distributions and to assign cluster membership. Briefly, the XD method fits the distribution of the elemental abundances as a sum of K Gaussian populations. Similar to K-means (Steinhaus 1956), the number of populations to fit is an input to the XD method and was fixed to two populations (FG and SG). This XD method was applied to each $\mathrm{GC}$ in our sample by using $[\mathrm{Mg} / \mathrm{Fe}]$, $[\mathrm{Al} / \mathrm{Fe}],[\mathrm{Si} / \mathrm{Fe}],[\mathrm{Ca} / \mathrm{Fe}]$, and $[\mathrm{Ti} / \mathrm{Fe}]$, as well as only $[\mathrm{Mg} / \mathrm{Fe}]$ and $[\mathrm{Al} / \mathrm{Fe}]$ (taking into account the individual abundance errors), and we found that $\mathrm{Mg}$ and $\mathrm{Al}$ drive the population membership in the most metal-poor $([\mathrm{Fe} / \mathrm{H}]<-1)$ GCs (see Mészáros et al. 2015 for more details). The exact $[\mathrm{Al} / \mathrm{Fe}]$ boundary between FG and SG stars is thus provided by the XD method and may differ slightly from one cluster to another. We note that (in the four GCs with SG-AGB stars identified, see below), this basically translates into FG and SG stars displaying roughly $[\mathrm{Al} / \mathrm{Fe}]<0.50 \mathrm{dex}$ (Al-poor) and $[\mathrm{Al} / \mathrm{Fe}] \geqslant 0.50 \mathrm{dex}$ (Al-rich), respectively. Thus, we combined our FG and SG star classification (mainly driven by the $\mathrm{Al}$ abundances) with the $U-(U-I), I-(U-I)$, and $V-(B-I)$ CMDs. We note that FGand SG-AGB stars display $[\mathrm{Al} / \mathrm{Fe}]<0.50 \mathrm{dex}$ (Al-poor) and $[\mathrm{Al} / \mathrm{Fe}] \geqslant 0.50 \mathrm{dex}$ (Al-rich), respectively. The only exception is the M2 AGB star 2M21331521-0049516, which displays a slightly lower $\mathrm{Al}$ abundance $([\mathrm{Al} / \mathrm{Fe}]=0.37 \mathrm{dex})$ and is classified as a SG star by the XD method.

The CMDs for four GCs $(\mathrm{M} 13:[\mathrm{Fe} / \mathrm{H}] \approx-1.53,81$ stars; M5: $[\mathrm{Fe} / \mathrm{H}] \approx-1.29,122$ stars; $\mathrm{M} 3:[\mathrm{Fe} / \mathrm{H}] \approx-1.50,55$ stars; and $\mathrm{M} 2:[\mathrm{Fe} / \mathrm{H}] \approx-1.65,18$ stars) contain SG Al-rich AGB stars (Figure 1). The AGB stars are clearly separated from those of the RGB in the $U-(U-I), I-(U-I)$, and $V-(B-I)$ CMDs (Figure 1). The only exceptions are: (i) four M13 AGB stars (the brightest ones near the tip of the RGB; all of them FG), which are not clearly separated from the RGB stars in the $V-(B-I)$ CMD but are in the $I-(U-I)$ and $U-(U-I)$ CMDs; (ii) two M3 AGB stars (both SG) that lie on the red RGB tail in the $U-(U-I)$ CMD but are clearly identified as AGB stars in the other two CMDs ${ }^{12}$; and (iii) one M2 AGB star (an FG one) that is not well separated from the RGBs in the $I-(U-I)$ and $V-(B-I)$ CMDs but is in the $U-(U-I)$ CMD. We identify a total of 4, 5, 3, and $2 \mathrm{SG}$ Al-rich AGB stars in M13, M5, M3, and M2, respectively. Table 1 lists the AGB stars (both FG and SG) identified together with some relevant observational information such as the APOGEE Al (and $\mathrm{O}$ where available) abundances and the $\mathrm{Na}$ and $\mathrm{O}$ abundances from the literature. ${ }^{13}$

\footnotetext{
${ }^{10}$ There is no significant differential reddening in the APOGEE GCs with available ground-based optical photometry, and the separation of AGB stars from RGB stars is easier.

${ }^{11} \mathrm{http}: / /$ github.com/jobovy/extreme-deconvolution

12 Our RGB/AGB identification is more conservative in M3 because the RGB is not so well defined as in the case of M13 and M5.

${ }^{13}$ There is only one $\mathrm{Na}$ I line $(1.639 \mu \mathrm{m})$ in the APOGEE $H$-band spectral range, which is too weak in the spectra of low-metallicity GC giant stars for reliable abundances to be derived.
} 

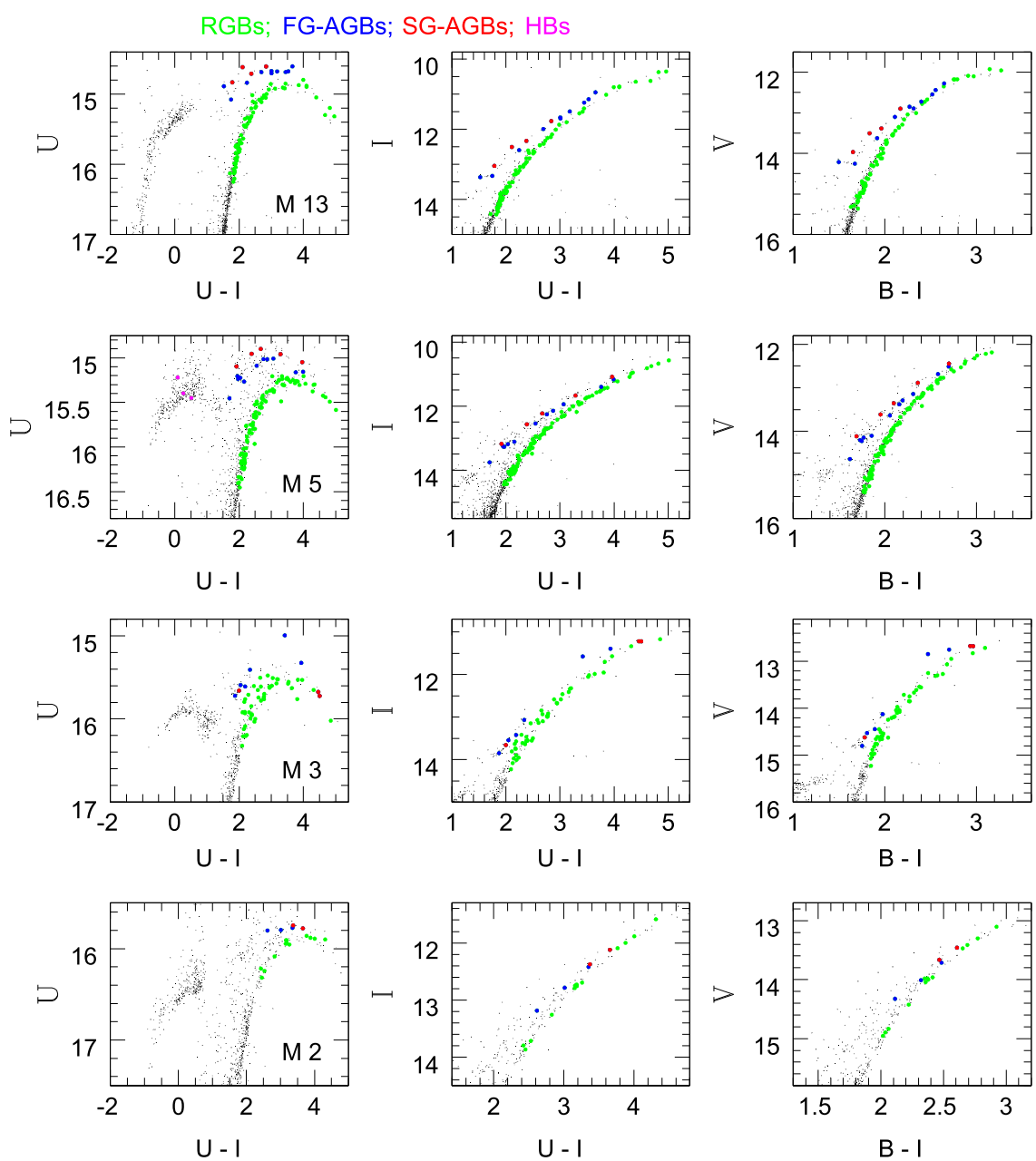

Figure 1. Color-magnitude (CMD) diagrams $U$ vs. ( $U-I$ ) (left panels), $I$ vs. ( $U-I)$ (middle panels), and $V$ vs. ( $B-I$ ) (right panels) for metal-poor GCs (from top to bottom: M13, M5, M3, and M2). Ground-based photometry for the cluster stars is indicated with black dots, while the RGB, FG-AGB, and SG-AGB stars observed by APOGEE are indicated with green, blue, and red dots, respectively. The three M5 stars marked with magenta dots (left panel) are HB stars.

The Al-O anticorrelation is clearly seen in our APOGEE data for all clusters (Figure 2) and the SG Al-rich AGB stars are among the most O-poor stars ${ }^{14}$, as expected. Only a few (5 out of 14) of the SG Al-rich AGB stars have Na abundances from optical spectroscopy available in the literature (Table 1). Remarkably, all of them are Na-rich $([\mathrm{Na} / \mathrm{Fe}] \sim 0.3-0.6$ dex; see Figure 3), supporting their identification as truly SG-AGB stars. Another indication of the Na-rich nature of the identified SG-AGB stars is offered by the $\left(V, C_{u, b, i}\right)$ pseudo-CMDs (where $C_{u, b, i}=(U-B)-(B-I)$; Monelli et al. 2013). It has been clearly shown by Monelli et al. (2013) that the $C_{u, b, i}$ index is very sensitive to any change in the relative distributions of CNO elements, and, since SG stars are N-rich/O-poor/Na-rich/ Al-rich with respect FG stars, it is a powerful tool for tracing the distribution of $\mathrm{FG} / \mathrm{SG}$ stars along the RGB and AGB evolutionary stages. In order to find an independent confirmation of present results, we show in Figure 4 the $\left(V, C_{u, b, i}\right)$

\footnotetext{
14 We use the most recent Asplund et al. (2005) solar abundance scale (e.g., $\mathrm{A}(\mathrm{O})=8.66)$, while earlier literature optical works generally use older solar abundance scales (e.g., Anders \& Grevesse 1989; Grevesse \& Sauval 1998) The mean offset of $\sim+0.2-0.3$ dex between the APOGEE and literature $\mathrm{O}$ abundances is just the consequence of using different solar abundance scales (Mészáros et al. 2015). Using older solar abundance scales, our APOGEE O abundances would be in good agreement with the literature; in particular the $[\mathrm{O} / \mathrm{Fe}]$ abundances in SG Al-rich AGB stars would be $\leqslant 0.0$.
}

pseudo-CMD of all the GCs in our sample. We find that (on average, with some exceptions) both RGB and AGB stars in our GC sample are separated in the $\left(V, C_{u, b, i}\right)$ pseudo-CMDs depending on their Al content (FG or SG). The SG Al-rich stars generally display higher values of the $C_{u, b, i}$ index, which corresponds to a population with higher $\mathrm{Na}$ content (Monelli et al. 2013). For example, at least two SG-AGB stars in M13 $(2 \mathrm{M} 16412975+3631563$ and $2 \mathrm{M} 16414398+3622338)$ lie in the region occupied by the most extreme Na-rich population defined by Monelli et al. (2013).

Finally, another interesting feature of Figure 1 is a hint for the presence of a splitting (i.e., different photometric sequences) along the AGB between the FG- and SG-AGB stars in M13 and M5. The number of stars, however, is small and this AGB splitting is not seen in M3 and M2, where we have observed even fewer stars. In the CMDs, the M13 and M5 SG-AGB stars seem to define bluer (and/or brighter) photometric sequences than the FG ones, as expected.

\section{DISCUSSION AND CONCLUSIONS}

The non-detection of SG-AGB stars in several metal-poor GCs (such as NGC 6752, M13, and M62) from previous optical spectroscopic surveys may be just coincidental (bias in the sample selections, small stellar samples) or due to the 
Table 1

AGB Stars in Metal-poor Globular Clusters ${ }^{\mathrm{a}}$

\begin{tabular}{|c|c|c|c|c|c|c|c|c|}
\hline 2MASS Name & $T_{\text {eff }}$ & $\log g$ & $\begin{array}{l}\text { Pop. }^{\text {b }} \\
\text { M13 }\end{array}$ & {$[\mathrm{Al} / \mathrm{Fe}]$} & {$[\mathrm{O} / \mathrm{Fe}]^{\mathrm{c}}$} & {$[\mathrm{Na} / \mathrm{Fe}]_{\text {Lit. }}{ }^{\mathrm{d}}$} & {$[\mathrm{O} / \mathrm{Fe}]_{\text {Lit. }}{ }^{\mathrm{d}}$} & $\overline{\text { References }^{\mathrm{e}}}$ \\
\hline $2 \mathrm{M} 16422126+3633533$ & 5136 & 2.59 & 1 & -0.39 & $\ldots$ & $0.20(0.26)$ & 0.69 & 1 \\
\hline $2 \mathrm{M} 16412975+3631563$ & 5173 & 2.67 & 2 & 0.82 & $\ldots$ & $\ldots$ & $\ldots$ & $\ldots$ \\
\hline $2 \mathrm{M} 16415003+3625105$ & 4698 & 1.65 & 1 & 0.26 & $\ldots$ & $0.30(0.18)$ & 0.25 & 1 \\
\hline $2 \mathrm{M} 16415543+3633266$ & 5024 & 2.33 & 2 & 0.75 & $\ldots$ & $\ldots$ & $\ldots$ & $\ldots$ \\
\hline $2 \mathrm{M} 16415024+3629431$ & 4909 & 2.08 & 2 & 0.59 & $\ldots$ & $\ldots$ & $\ldots$ & $\ldots$ \\
\hline $2 \mathrm{M} 16415452+3626289$ & 5376 & 3.23 & 1 & 0.42 & $\ldots$ & $\ldots$ & $\ldots$ & $\ldots$ \\
\hline $2 \mathrm{M} 16413082+3630130$ & 4950 & 2.16 & 1 & 0.04 & $\ldots$ & 0.05 & 0.29 & 6 \\
\hline $2 \mathrm{M} 16414398+3622338$ & 4606 & 1.51 & 2 & 0.72 & $\ldots$ & $0.29(0.13)$ & 0.10 & 1 \\
\hline $2 \mathrm{M} 16420085+3623338$ & 4594 & 1.45 & 1 & 0.16 & $\ldots$ & $0.22(0.06)$ & 0.32 & 1 \\
\hline $2 \mathrm{M} 16412408+3625306$ & 4366 & 1.08 & 1 & 0.20 & 0.60 & $0.00(-0.16)$ & 0.14 & 1 \\
\hline $2 \mathrm{M} 16412709+3628002$ & 4366 & 1.08 & 1 & -0.25 & 0.59 & -0.09 & 0.30 & 6 \\
\hline $2 \mathrm{M} 16413961+3627381$ & 4337 & 1.03 & 1 & -0.14 & 0.58 & $0.01(-0.15)$ & 0.38 & 1 \\
\hline $2 \mathrm{M} 16414966+3627104$ & 4512 & 1.32 & 1 & -0.30 & 0.60 & $-0.26(-0.42)$ & 0.46 & 1 \\
\hline \multirow[t]{2}{*}{$2 \mathrm{M} 16414517+3628132$} & 4435 & 1.17 & 1 & -0.15 & 0.54 & $0.25(0.09)$ & 0.34 & 1 \\
\hline & & & M5 & & & & & \\
\hline $2 \mathrm{M} 15184048+0210446$ & 5499 & 3.58 & 1 & -0.28 & $\ldots$ & $\ldots$ & $\ldots$ & $\ldots$ \\
\hline $2 \mathrm{M} 15180831+0158530$ & 4922 & 2.30 & 1 & 0.10 & $\ldots$ & 0.35 & 0.30 & 2 \\
\hline $2 \mathrm{M} 15183957+0205018$ & 5071 & 2.63 & 2 & 0.50 & $\ldots$ & $\ldots$ & $\ldots$ & $\ldots$ \\
\hline $2 \mathrm{M} 15184022+0213278$ & 4966 & 2.41 & 1 & -0.26 & $\ldots$ & $\ldots$ & $\ldots$ & $\ldots$ \\
\hline $2 \mathrm{M} 15175224+0208026$ & 5078 & 2.63 & 1 & 0.24 & $\ldots$ & $\ldots$ & $\ldots$ & $\ldots$ \\
\hline $2 \mathrm{M} 15185731+0203077$ & 5067 & 2.63 & 1 & 0.26 & $\ldots$ & $\ldots$ & $\ldots$ & $\ldots$ \\
\hline $2 \mathrm{M} 15183638+0208507$ & 4842 & 2.12 & 2 & 0.84 & $\ldots$ & $\ldots$ & $\ldots$ & $\ldots$ \\
\hline $2 \mathrm{M} 15180987+0210088$ & 4810 & 2.06 & 1 & -0.05 & $\ldots$ & -0.04 & 0.56 & 2 \\
\hline $2 \mathrm{M} 15183575+0204297$ & 6155 & 3.87 & 2 & 1.06 & $\ldots$ & $\ldots$ & $\ldots$ & $\ldots$ \\
\hline $2 \mathrm{M} 15185515+0214337$ & 4639 & 1.73 & 1 & -0.15 & $\ldots$ & -0.01 & 0.31 & 2 \\
\hline $2 \mathrm{M} 15182435+0201574$ & 4507 & 1.53 & 2 & 0.90 & 0.03 & $0.41(0.25)$ & -0.11 & 3 \\
\hline $2 \mathrm{M} 15183738+0206079$ & 4207 & 0.96 & 2 & 0.50 & 0.29 & 0.29 & 0.28 & 2 \\
\hline $2 \mathrm{M} 15174702+0204519$ & 4391 & 1.30 & 1 & -0.10 & 0.44 & -0.08 & 0.47 & 2 \\
\hline $2 \mathrm{M} 15182014+0203321$ & 4533 & 1.53 & 1 & 0.09 & 0.32 & $0.20(0.04)$ & 0.13 & 3 \\
\hline $2 \mathrm{M} 15184540+0204302$ & 4283 & 1.10 & 1 & 0.15 & 0.41 & 0.26 & 0.49 & 2 \\
\hline \multirow[t]{2}{*}{$2 \mathrm{M} 15184139+0206004$} & 4306 & 1.15 & 1 & 0.27 & 0.51 & $0.52(0.33)$ & 0.35 & 3 \\
\hline & & & M3 & & & & & \\
\hline $2 \mathrm{M} 13423482+2826148$ & 4904 & 2.13 & 1 & -0.24 & $\ldots$ & -0.19 & $\ldots$ & 4 \\
\hline $2 \mathrm{M} 13414871+2820024$ & 5011 & 2.36 & 2 & 0.77 & $\ldots$ & $\ldots$ & $\ldots$ & $\ldots$ \\
\hline $2 \mathrm{M} 13421373+2821154$ & 5032 & 2.40 & 1 & -0.19 & $\ldots$ & 0.08 & $\ldots$ & 4 \\
\hline $2 \mathrm{M} 13422197+2828408$ & 4700 & 1.71 & 1 & -0.12 & $\ldots$ & -0.19 & $\ldots$ & 4 \\
\hline $2 \mathrm{M} 13425083+2827576$ & 4880 & 2.08 & 1 & -0.13 & $\ldots$ & -0.17 & $\ldots$ & 4 \\
\hline $2 \mathrm{M} 13421712+2822137$ & 4418 & 1.22 & 1 & -0.08 & 0.58 & 0.05 & $\ldots$ & 4 \\
\hline $2 \mathrm{M} 13414576+2824597$ & 4315 & 1.00 & 1 & -0.15 & 0.67 & -0.26 & 0.36 & 4,5 \\
\hline $2 \mathrm{M} 13415152+2823224$ & 4015 & 0.50 & 2 & 0.78 & 0.12 & 0.57 & -0.24 & 6 \\
\hline \multirow[t]{2}{*}{$2 \mathrm{M} 13421086+2823465$} & 4047 & 0.56 & 2 & 0.78 & 0.23 & $0.61(0.42)$ & -0.05 & 5 \\
\hline & & & M2 & & & & & \\
\hline 2M21332545-0047056 & 4710 & 1.63 & 1 & -0.25 & $\ldots$ & $\ldots$ & $\ldots$ & $\ldots$ \\
\hline 2M21332531-0052511 & 4531 & 1.31 & 1 & -0.38 & 0.65 & $\ldots$ & $\ldots$ & $\ldots$ \\
\hline 2M21331521-0049516 & 4554 & 1.35 & 2 & 0.37 & $\ldots$ & $\ldots$ & $\ldots$ & $\ldots$ \\
\hline 2M21333432-0051285 & 4436 & 1.14 & 1 & -0.34 & 0.63 & $\ldots$ & $\ldots$ & $\ldots$ \\
\hline 2M21332527-0049386 & 4098 & 0.53 & 2 & 0.68 & -0.02 & $\cdots$ & $\cdots$ & $\cdots$ \\
\hline
\end{tabular}

Notes.

${ }^{a}$ Effective temperatures $\left(T_{\text {eff }}\right)$, surface gravities $(\log g)$, Al, and O abundances from Mészáros et al. (2015).

${ }^{\mathrm{b}}$ Population: 1 and 2 are first-generation and second-generation, respectively.

${ }^{c}[\mathrm{O} / \mathrm{Fe}]$ abundances from Mészáros et al. (2015); only available for stars with $T_{\text {eff }}$ below $4520 \mathrm{~K}$. Note that our APOGEE [O/Fe] abundances are $\sim+0.2-0.3$ dex sistematically higher than the literature values because of the use of different solar abundance scales (Mészáros et al. 2015).

${ }^{\mathrm{d}} \mathrm{Na}$ and $\mathrm{O}$ abundances in the literature (from high-resolution optical spectra). The Na abundances corrected for NLTE effects according to Gratton et al. (1999) are given. Johnson \& Pilachowski (2012) and Ivans et al. (2001) did not report the measured Na I 6154 A equivalent widths (EWs) for the M 13 and M5 AGB stars, respectively, while Cavallo \& Nagar (2000) report an EW of 34 mA for one M3 AGB star in our sample. Thus, for these stars we assumed an average EW of $30 \mathrm{~m} \AA$ (e.g., the EWs range in M13 giant stars is $\sim 10-50 \mathrm{~m} \AA$; Cohen \& Meléndez (2005)) and $[\mathrm{Fe} / \mathrm{H}]=-1.50$ to make conservative NLTE corrections and we list also the non-corrected $\mathrm{Na}$ abundances in parenthesis.

${ }^{\mathrm{e}}$ Reference for the $\mathrm{O}$ and $\mathrm{Na}$ abundances from high-resolution optical spectra.

References. (1) Johnson \& Pilachowski (2012), (2) Lai et al. (2011), (3) Ivans et al. (2001), (4) Johnson et al. (2005), (5) Cavallo \& Nagar (2000), (6) Sneden et al. (2004). 

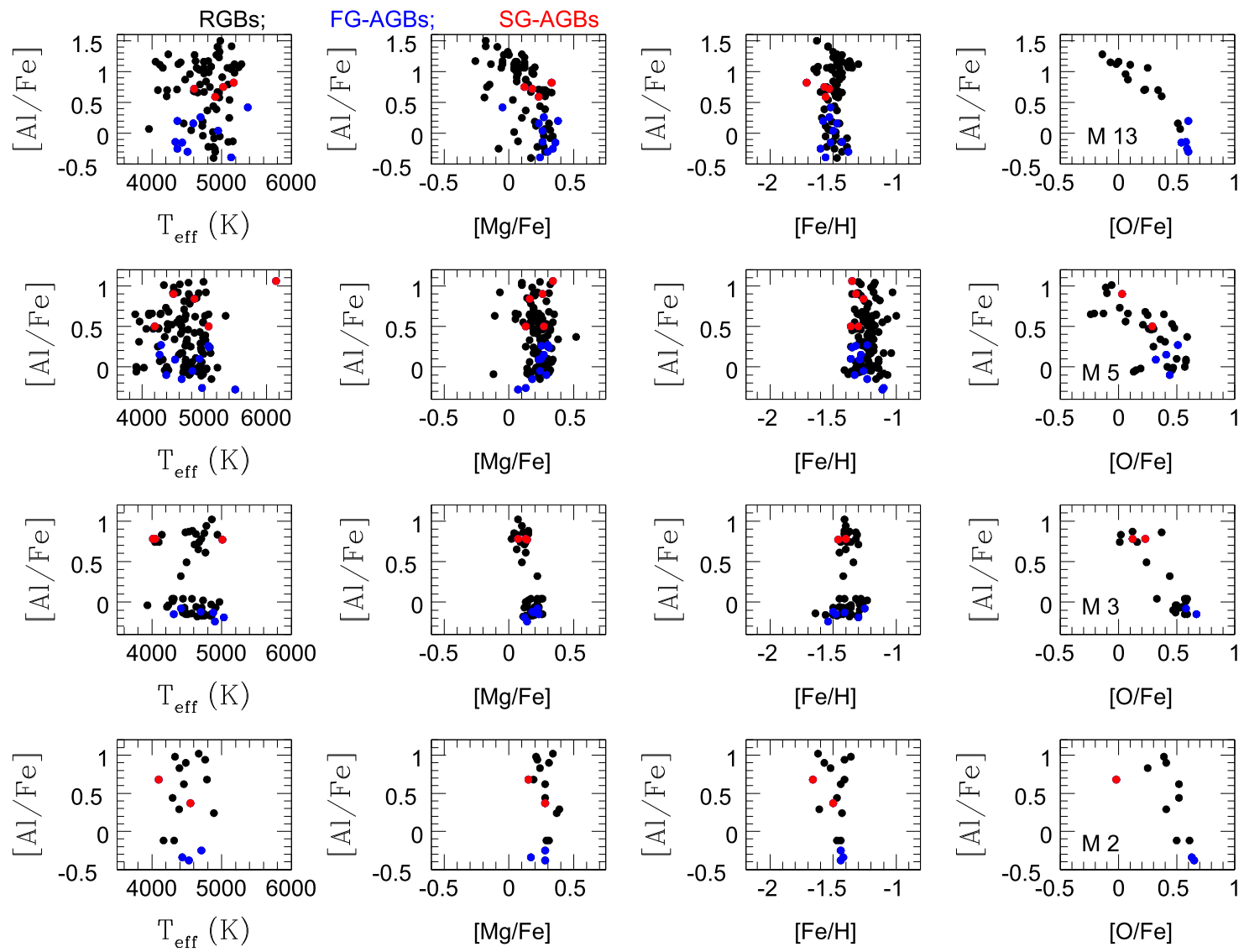

Figure 2. [Al/Fe] for our APOGEE sample of FG- and SG-AGB stars (blue and red dots, respectively) in metal-poor GC stars (from top to bottom: M13, M5, M3, and $\mathrm{M} 2$ ) shown against (from left to right) stellar effective temperature $T_{\text {eff }},[\mathrm{Mg} / \mathrm{Fe}],[\mathrm{Fe} / \mathrm{H}]$, and $[\mathrm{O} / \mathrm{Fe}]$. The APOGEE abundances are precise to the $\sim 0.1$ dex level. For comparison, the RGB stars (black dots) observed by APOGEE are also displayed. Note that the spread in $[\mathrm{Fe} / \mathrm{H}]$ is bigger in RGB than in AGB stars because of the presence of warm stars, which lead to bigger uncertainties (consequentely $[\mathrm{Al} / \mathrm{Fe}]$ is determined with higher accuracy in AGB than in RGB stars).
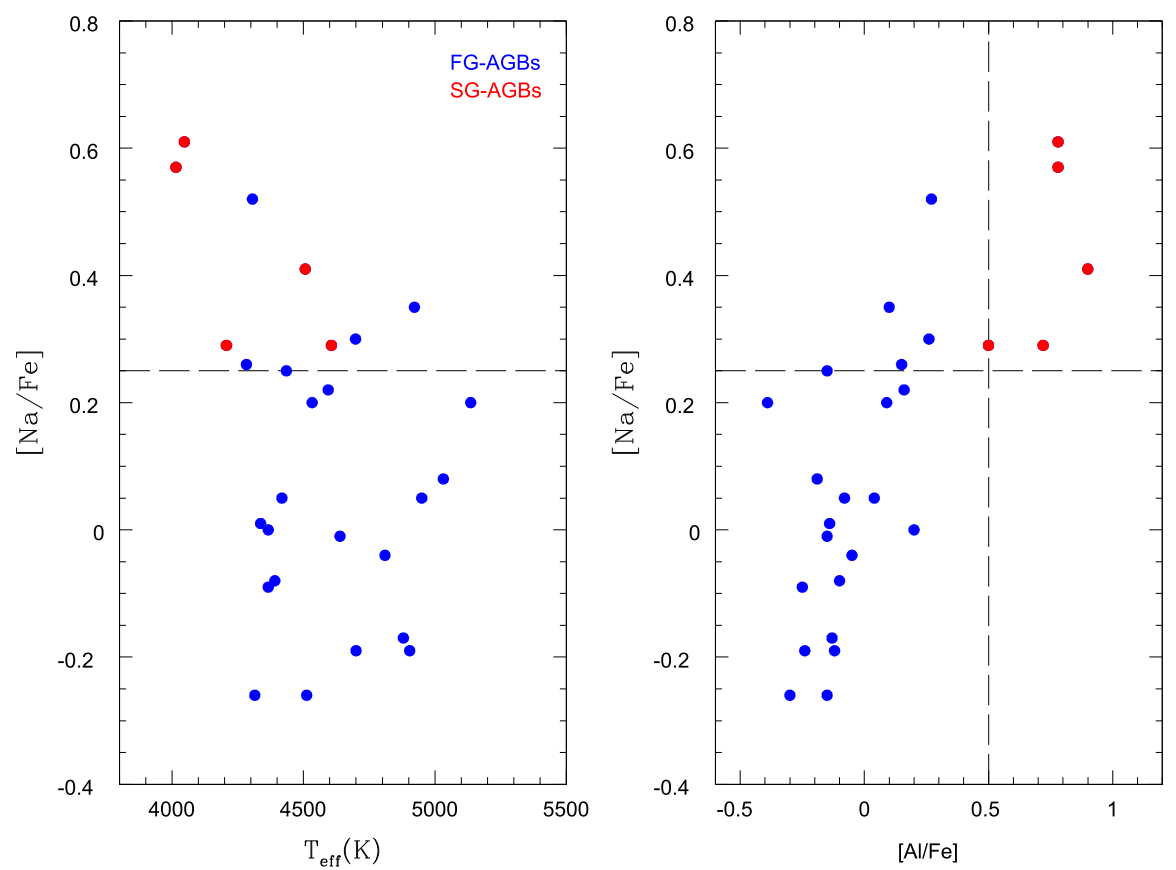

Figure 3. Literature $[\mathrm{Na} / \mathrm{Fe}]$ abundances (corrected for NLTE) for our APOGEE sample of FG- and SG-AGB stars (blue and red dots, respectively) shown against stellar effective temperature $T_{\text {eff }}$ (left panel) and $\mathrm{Al}$ abundances (right panel). The horizontal and vertical lines mark $[\mathrm{Na} / \mathrm{Fe}]=+0.25 \mathrm{dex}$ and $[\mathrm{Al} / \mathrm{Fe}]=+0.50 \mathrm{dex}$, respectively and separate the $\mathrm{FG}-\mathrm{AGBs}$ from the $\mathrm{SG}$ ones. The $\mathrm{Na}$ limit is set to $[\mathrm{Na} / \mathrm{Fe}]=0.25 \mathrm{dex}$, corresponding to the average upper limit for the $[\mathrm{Na} / \mathrm{Fe}]$ value for FG field stars in the metallicity range covered by the GCs in our sample (see e.g., Carretta 2013). 

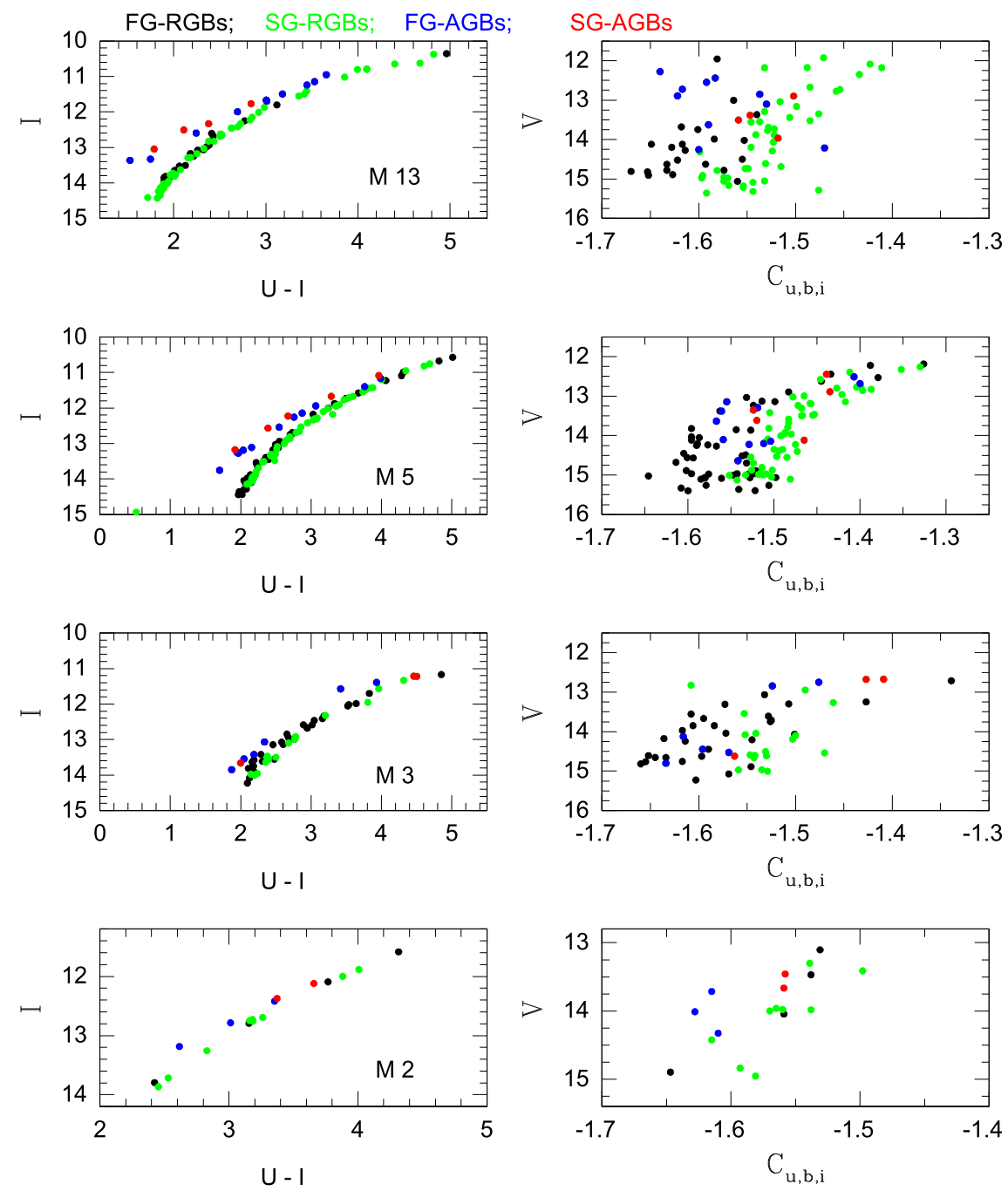

Figure 4. Color-magnitude (CMD) diagrams $I$ vs. $(U-I)$ (left panels) and the $\left(V, C_{u, b, i}\right)$ pseudo-CMDs (right panel) for the metal-poor GCs (from top to bottom: M13, M5, M3, and M2) observed by APOGEE. FG- and SG-AGB stars are marked (blue and red dots, respectively). For comparison, the FG- and SG-RGB stars (black and green dots, respectively) are also displayed.

non-use of recent (and precise) optical photometry and appropriate combinations of several CMDs for efficient RGB/AGB separation. For example, we have several M13 AGB stars (9 FG and $1 \mathrm{SG}$; see Table 1) in common with Johnson \& Pilachowski (2012). These authors used the $V$ $(V-K)$ CMD (with coordinates and $V$ photometric data by Cudworth \& Monet 1979) to separate the RGB from the AGB stars. They are not able to efficiently discriminate the RGB from the AGB (especially near the tip of the RGB) because the width of the RGB in their $V-(V-K)$ CMD is much wider than ours (when using our more recent photometric data). Indeed, the only M13 SG-AGB star (2M16414398+3622338; Table 1) in common with us was wrongly classified by these authors as an RGB star. Our AGB identifications in M5 are fully consistent with the previous optical studies using recent photometric data. The previous optical works in M3 did not attempt any RGB/AGB separation from appropriate combinations of several CMDs, while no M2 AGB star in our sample has been previously studied (see Table 1).

The lack of Na-rich SG-AGB stars in NGC 6752 (Campbell et al. 2013) is puzzling (also in M62 but only six AGB stars were analyzed; Lapenna et al. 2015). Here we report for the first time SG-AGB stars in the GC M13; a twin of NGC 6752 with very similar HB morphology, metallicity, and age. An alternative explanation for the Na-poor character of all AGBs surveyed in NGC 6752 (as well as for the previous nondetection of Na-rich SG-AGBs in several metal-poor GCs) is the fact that NLTE effects in AGB stars may be larger than in RGB stars. This would underestimate more severely the correct $\mathrm{Na}$ abundances in the AGB stars (Lapenna et al. 2015 and references therein). Higher NLTE effects in AGB stars are suggested by the differences (up to $\sim 0.1-0.2 \mathrm{dex}$ ) in the $\mathrm{Fe}$ (and Ti) abundances measured from neutral and single-ionized lines in the AGB stars, which otherwise are negligible in the RGB stars (e.g., Ivans et al. 2001; Lapenna et al. 2015). For example, the $\mathrm{Fe}$ abundances derived from optical neutral lines in AGB stars are systematically $\sim 0.1-0.2$ dex lower than in the RGB stars. As we mentioned above, the APOGEE abundances are measured from neutral lines and we find no significant differences for the $\mathrm{Fe}(\mathrm{Al}, \mathrm{Mg}, \mathrm{O})$ abundances in the AGB and RGB stars (see Figure 2). This confirms that the $H$-band spectral lines are formed deeper in the atmosphere and NLTE effects on the neutral lines of $\mathrm{Fe}, \mathrm{Al}, \mathrm{Mg}$, etc. are less severe than in the optical domain. The $H$-band thus opens up a new (and safer) window to systematically study the AGB and RGB stellar generations in Galactic GCs. 
In conclusion, our results provide plain evidence of the fact that SG stars are present along the AGB of metal-poor Galactic GCs. This supports the present generation of canonical HB stellar models in terms of their capability to properly reproduce the observed distribution of FG and SG stars during both the core and shell He-burning phases.

D.A.G.H. was funded by the Ramón y Cajal fellowship number RYC-2013-14182. D.A.G.H. and O.Z. acknowledge support provided by the Spanish Ministry of Economy and Competitiveness (MINECO) under grant AYA-2014-58082-P. Sz.M. has been supported by the János Bolyai Research Scholarship of the Hungarian Academy of Sciences. S.C. acknowledges partial financial support from PRIN-INAF2014 (PI: S. Cassisi) and the Instituto de Astrofísica de Canarias for inviting him as a Severo Ochoa visitor during 2015 April to June when part of this work was done. S.L. acknowledges partial support from PRIN-MIUR 2010-2011 (PI: F. Matteucci). Funding for SDSS-III has been provided by the Alfred P. Sloan Foundation, the Participating Institutions, the National Science Foundation, and the U.S. Department of Energy Office of Science. The SDSS-III web site is http://www.sdss3.org/. SDSS-III is managed by the Astrophysical Research Consortium for the Participating Institutions of the SDSS-III Collaboration including the University of Arizona, the Brazilian Participation Group, Brookhaven National Laboratory, University of Cambridge, Carnegie Mellon University, University of Florida, the French Participation Group, the German Participation Group, Harvard University, the Instituto de Astrofísica de Canarias, the Michigan State/Notre Dame/JINA Participation Group, Johns Hopkins University, Lawrence Berkeley National Laboratory, Max Planck Institute for Astrophysics, New Mexico State University, New York University, Ohio State University, Pennsylvania State University, University of Portsmouth, Princeton University, the Spanish Participation Group, University of Tokyo, University of Utah, Vanderbilt University, University of Virginia, University of Washington, and Yale University.

\section{REFERENCES}

Anders, E., \& Grevesse, N. 1989, GeCoA, 53, 197

Asplund, M., Grevesse, N., \& Sauval, A. J. 2005, in ASP Conf. Ser. 336, Cosmic Abundances as Records of Stellar Evolution and Nucleosynthesis, ed. T. G. Barnes III \& F. N. Bash (San Francisco, CA: ASP), 25

Campbell, S. W., D’Orazi, V., Yong, D., et al. 2013, Natur, 498, 198 Carretta, E. 2013, A\&A, 557, A128

Carretta, E., Bragaglia, A., Gratton, R., \& Lucatello, S. 2009, A\&A, 505, 139

Cassisi, S., \& Salaris, M. 2013, Old Stellar Populations: How to Study the Fossil Record of Galaxy Formation (London: Wiley-VCH)

Cassisi, S., Salaris, M., Pietrinferni, A., Vink, J. S., \& Monelli, M. 2014, A\&A, 571, A81

Cavallo, R. M., \& Nagar, N. M. 2000, AJ, 120, 1364

Charbonnel, C., Chantereau, W., Decressin, T., Meynet, G., \& Schaerer, D. 2013, A\&A, 557, L17

Cohen, J. G., \& Meléndez, J. 2005, AJ, 129, 303

Cudworth, K. M., \& Monet, D. G. 1979, AJ, 84, 774

Gratton, R. G., Bonifacio, P., Bragaglia, A., et al. 2001, A\&A, 369, 87

Gratton, R. G., Carretta, E., \& Bragaglia, A. 2012, A\&ARv, 20, 50

Gratton, R. G., Carretta, E., Eriksson, K., \& Gustafsson, B. 1999, A\&A, 350,955

Gratton, R. G., D'Orazi, V., Bragaglia, A., Carretta, E., \& Lucatello, S. 2010, A\&A, 522, A77

Grevesse, N., \& Sauval, A. J. 1998, SSRv, 85, 161

Ivans, I. I., Kraft, R. P., Sneden, C., et al. 2001, AJ, 122, 1438

Johnson, C. I., Kraft, R. P., Pilachowski, C. A., et al. 2005, PASP, 117,1308

Johnson, C. I., McDonald, I., Pilachowski, C. A., et al. 2015, AJ, 149, 71

Johnson, C. I., \& Pilachowski, C. A. 2012, ApJL, 754, L38

Lai, D. K., Smith, G. H., Bolte, M., et al. 2011, AJ, 141, 62

Lapenna, E., Mucciarelli, A., Ferraro, F. R., et al. 2015, ApJ, 813, 97

Majewski, S. R., Schiavon, R. P., Allende Prieto, C., et al. 2015, AJ, submitted (arXiv: 1509.05420)

Marino, A. F., Milone, A. P., Przybilla, N., et al. 2014, MNRAS, 437, 1609

Mészáros, Sz., Martell, S. L., Shetrone, M., et al. 2015, AJ, 149, 153

Milone, A. P., Marino, A. F., Dotter, A., et al. 2014, ApJ, 785, 21

Milone, A. P., Piotto, G., Bedin, R. L., et al. 2012, ApJ, 744, 58

Monelli, M., Milone, A. P., Stetson, P. B., et al. 2013, MNRAS, 431, 2126

Norris, J., Cottrell, P. L., Freeman, K. C., \& Da Costa, G. S. 1981, ApJ, 244,205

Piotto, G., Milone, A. P., Anderson, J., et al. 2012, ApJ, 760, 39

Sneden, C., Kraft, R. P., Guhathakurta, P., Peterson, R. C., \& Fulbright, J. P. 2004, AJ, 127, 2162

Steinhaus, H. 1956, Bull. Acad. Polon. Sci., 4, 801

Stetson, P. B., Braga, V. F., Dall'Ora, M., et al. 2014, PASP, 126, 521 